\title{
MDRD, CKD-Epi and Creatinine Clearance with 24-Hour Urine Collection Results in Patients with Chronic Kidney Disease
}

\author{
Siti Nurul Hapsari, Leonita Anniwati \\ Department of Clinical Pathology, Faculty of Medicine, Airlangga University/Dr. Soetomo Hospital, Surabaya, Indonesia. E-mail: \\ nu.neemo@gmail.com
}

\begin{abstract}
Kidney disease is a global public health problem, affecting over 750 million people worldwide. Glomerular Filtration Rate (GFR), which is calculated by measuring the creatinine clearance with 24-hour urine collection (CC) can be inaccurate due to improper urine collection, causing the need for an easier and accurate method of calculation. This study was an observational analytical cross-sectional research using consecutive retrospective sampling. Samples were data of patients with Chronic Kidney Disease (CKD) who underwent CC test at the Clinical Pathology Laboratory of the Dr. Soetomo Hospital Surabaya during September-October 2018. Data were compared with the results of Cockcroft-Gault (CG), MDRD, and CKD-Epi formula, and were analyzed using the one-sample Kolmogorov-Smirnov test, paired T-test, and Wilcoxon Signed Rank test. Correlation of CC results with CG, MDRD, and CKD-Epi results was tested with Spearman's rho and Bland Altman test. The difference test of CC with CG, MDRD, and CKD-Epi showed results of $(p=0.000),(p=0.194)$, and $(p=0.468)$, respectively. There were significant differences between CC compared to CG, but not MDRD and CKD-Epi. There was a moderate correlation between CG, MDRD, CKD-Epi, and CC with $r=0.529 ; 0.448$, and 0.463 , respectively. The most compatible formula was CKD-Epi. The measurement of GFR with CC correlated with CG, MDRD, and CKD-Epi; therefore, they could be used as an alternative method to calculate GFR. Further experiments using an exogenous marker should be performed to determine a suitable eGFR formula according to the degree of damage to the kidney.
\end{abstract}

Keywords: Chronic kidney disease, glomerular filtration rate, creatinine clearance, eGFR

\section{INTRODUCTION}

Chronic Kidney Disease (CKD) is a group of the pathophysiological process that is related to a disorder in kidney function and progressive decrease of Glomerular Filtration Rate (GFR). End-Stage Renal Disease (ESRD) is a CKD stage in which the accumulation of toxins, fluid, and electrolytes are not normally excreted by the kidney and results in uremic syndrome. ${ }^{1}$

Kidney disease is a global public health problem, affecting over 750 million people worldwide. The burden of kidney disease varies substantially across the world. ${ }^{2}$ The increase of death due to CKD increases from 8 in every 100,000 people to 13 in 100,000 (from $1990-2013$ ). ${ }^{3}$

The measurement of kidney function and the changes after an intervention is limited in the calculation of GFR, the kidney blood flow, and proteinuria estimation. The glomerular filtration rate is the flow rate of filtered substances in plasma or the clearance rate of substances in the blood through the glomerulus expressed in millimeters per minute. No endogenous marker is ideal for GFR calculation.
Inulin is considered the reference method for calculating GFR; however, it is inconvenient because its measurement must be carried out in specialized centers, and the assays are difficult. Therefore, another method is needed. ${ }^{4}$

Creatinine is the by-product of creatinine phosphate in muscle and is produced at a constant rate by the body. Creatinine is not an ideal filtration marker like inulin because it is not merely filtrated in the glomerulus, but it is also secreted in the tubulus. Creatinine secretion by the tubulus is not constant for certain individuals; there is a difference between males, females, and children, and it is also influenced by the ingestion of red meat. Serum creatinine level is usually used to measure kidney function. This parameter is determined by renal elimination and creatinine production. The quantitative measurement of this protein marker is used to estimate the degree of damage and the damaged site in the glomerular neuron. This can calculate the progress of kidney disease. ${ }^{4}$

The GFR calculation by creatinine clearance can be inaccurate if the 24-hour urine collection is not properly handled; therefore, an easier and more accurate method is needed. 
This study was performed to determine the compatibility of the results of glomerular filtration rate calculation using the Cockroft-Gault (CG) formula, Modification of Diet in Renal Disease (MDRD), Chronic Kidney Disease Epidemiology Collaboration (CKD-Epi), and creatinine clearance using 24-hour urine collection in CKD at Dr. Soetomo Hospital, Surabaya.

\section{METHODS}

This study was observational, experimental research using a cross-sectional design with consecutive retrospective sampling. The samples were data of patients who measured their creatinine clearance with the 24-hour collection method (CC) from September to October 2018 at the Clinical Pathology Laboratorium of Dr. Soetomo Hospital, compared with GFR calculated with the CG, MDRD, and CKD-Epi method (Table 1).

Data were then analyzed using IBM SPSS version 22.2. One sample Kolmogorov-Smirnov test was used for the normality test and paired T-test was used as a different test for data with normal distribution, and the Wilcoxon Signed-Rank test was used for data with the abnormal distribution. The correlation of CC with CG, MDRD, and CKD-Epi was determined with Spearman's rho and Bland Altman test with a $p<0.05$ and confidence interval of $95 \%$.

\section{RESULTS AND DISCUSSION}

This study used data of clearance creatinine using a 24-hour collection method of chronic kidney disease patients during October and September 2018. From 520 subjects with creatinine clearance data, 20 subjects were excluded due to incomplete data or no CKD reported in those patients; therefore, total subjects were 500 . The characteristics of the subjects can be seen in Table 2 .

It can be seen from Table 2 that the male ratio in this study was greater than the female $(71 \%)$. The mean age was 50.3 years old, with a mean value of CC of $84.08 \mathrm{ml} / \mathrm{min} / 1.73 \mathrm{~m}^{2}$. Wilcoxon Ranks test is used to analyze the difference between CC, CG, and due to the abnormal distribution of the data with a $p$-value of 0.000 for CG and p-value of 0.194 for MDRD, suggesting a significant difference between the results of CC and CG; but no significant difference was found between CG results and MDRS. In addition, a paired T-test was used for the differential analysis between CC and CKD-Epi due to the normal distribution of data. The paired T-test showed a $p$-value of 0.468 , suggesting no significant differences between CC and CKD-Epi results. Table 3 shows the results of the differential analysis of each method compared to CC.

Table 1. Creatinine clearance test ${ }^{5}$

\begin{tabular}{|c|c|}
\hline Method & Formula \\
\hline $\mathrm{CC}$ & $\begin{array}{l}\text { (24-hour urine volume } / 1440) \times(\text { urine creatinine/serum } \\
\text { creatinine) } \times(1.73 / \text { body surface area) }\end{array}$ \\
\hline CG & $\left\{((140\right.$-age $) \times$ weight $\left.) /\left(72 \mathrm{~S}_{\mathrm{Cr}}\right)\right\} \times 0.85$ if female \\
\hline MDRD & $\begin{array}{l}186 \times(\mathrm{S})^{-1.154} \times(\text { age })^{-0.203} \times(0.742 \mathrm{Cr} \\
\text { if female }) \times(1.210 \text { if African American })\end{array}$ \\
\hline CKD-Epi & $\begin{array}{l}141 \times \min \{\text { creatinine } / \mathrm{k}, 1\}^{\alpha} \times \max \{\text { creatinine } / \mathrm{l}, 1\}^{-1.209} \times \\
0.993^{\mathrm{age}} \times 1.018 \text { [if female] } \times 1.159 \text { [if African] }\end{array}$ \\
\hline
\end{tabular}

Table 2. Subjects characteristics

\begin{tabular}{ll}
\hline Characteristics & Mean \\
\hline Male, $\mathrm{n}: 355(71 \%)$ & \\
Female, $\mathrm{n}: 145(29 \%)$ & \\
Age $($ years old $)$ & $50.3 \pm 11.44$ \\
BSA $\left(\mathrm{kg} / \mathrm{m}^{2}\right)$ & $1.84 \pm 6.55$ \\
Height $(\mathrm{cm})$ & $159,14 \pm 10.54$ \\
Weight $(\mathrm{kg})$ & $55.81 \pm 13.08$ \\
Urine volume $(\mathrm{cc})$ & $2311,66 \pm 1402,30$ \\
Serum creatinine $(\mathrm{mg} / \mathrm{dL})$ & $1.23 \pm 1.48$ \\
Urine creatinine $(\mathrm{mg} / \mathrm{dL})$ & $67.53 \pm 58.28$ \\
Clearance creatinine $\left(\mathrm{mL} /\right.$ min per $\left.1.73 \mathrm{~m}^{2}\right)$ & $84.08 \pm 39.36$ \\
\hline
\end{tabular}


The correlation of CC with CG, MDRD, and CKD-Epi was analyzed using Spearman's rho test because the distribution of the data was not normal, and plotted using Bland Altman, with the results as follows:

Table 3. Result of differential analysis

\begin{tabular}{clll}
\hline Parameter & Mean \pm SD & p & Interpretation \\
\hline CC - CG & $84.08 \pm 39,356$ & 0.000 & Significant difference \\
CC & $85.18 \pm 28,982$ & & \\
CG & & 0.194 & $\begin{array}{l}\text { No significant } \\
\text { difference }\end{array}$ \\
CC - MDRD & $84.08 \pm 39,356$ & & \\
CC & $83.22 \pm 34.43$ & & No significant \\
MDRD & & 0.468 & difference \\
CC - CKD-Epi & $84.08 \pm 39,356$ & & \\
CC & $72.13 \pm 29.71$ & & \\
CKD-Epi & & & \\
\hline
\end{tabular}
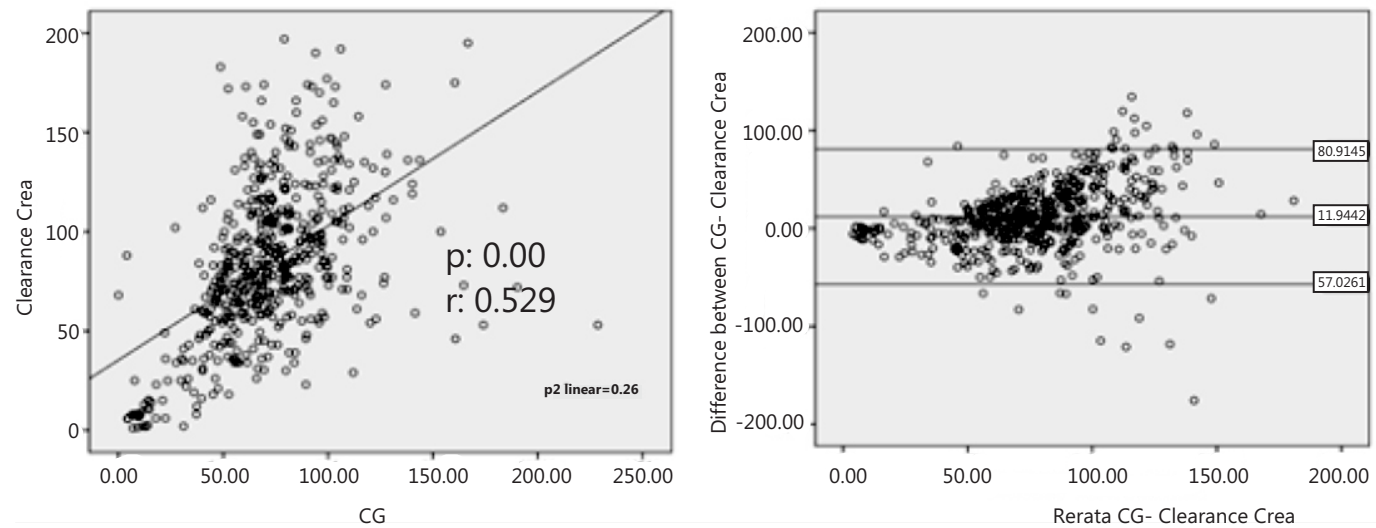

Figure 1. Compatibility between CC and CG
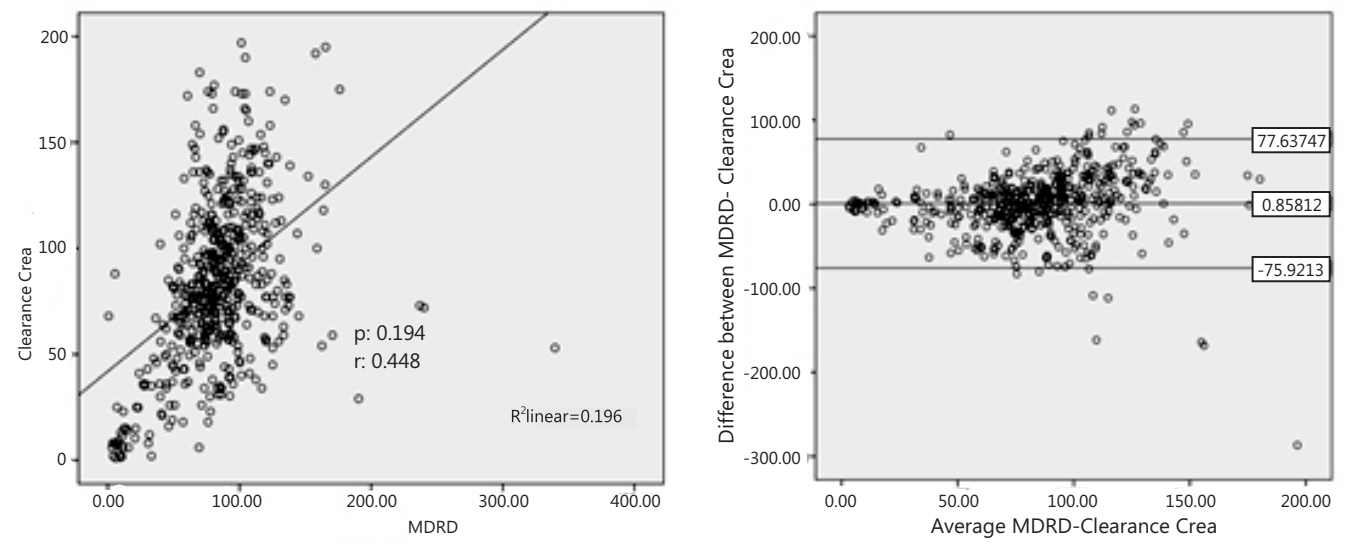

Figure 2. Compatibility between CC and MDRD
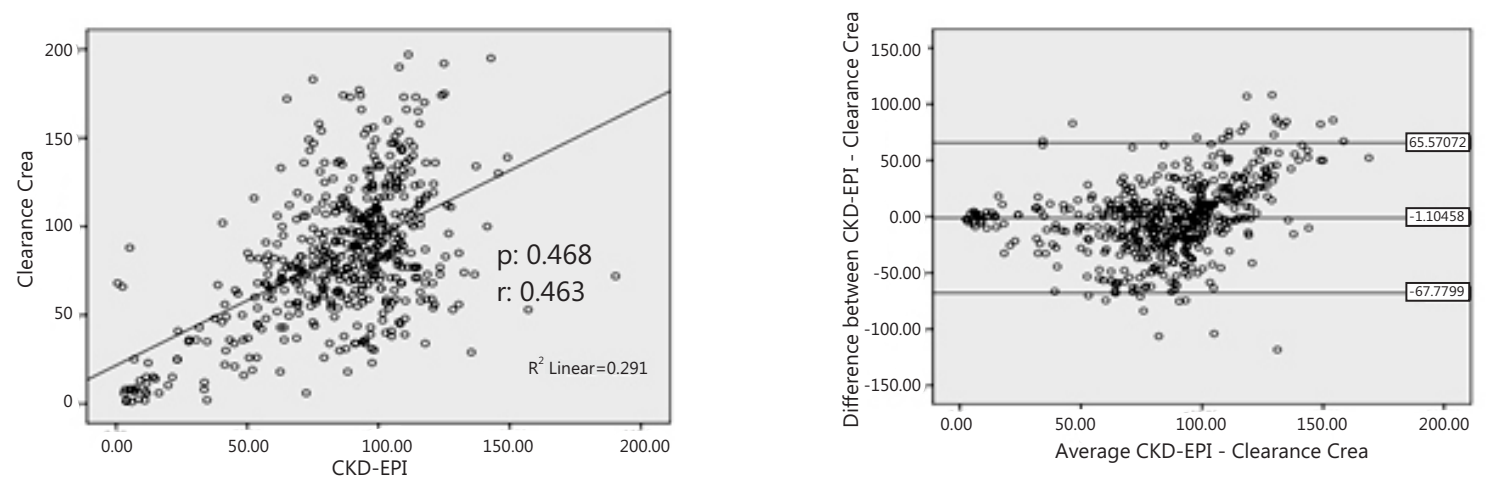

Figure 3. Compatibility between ACC and CKD-Epi 
It can be seen in Fig. 1, 2, and 3 that CG, MDRD, and CKD-Epi were correlated with CC results with $r$ of $0.529 ; 0.448$, and 0.463 ( $p$ 0.00), respectively. The formula, which had the most correlation with CC was CKD-Epi, followed by CG.

Damage of the kidney is a big mortality risk in many populations; therefore, an accurate method to measure kidney damage is highly needed. Measured GFR (mGFR) is the gold standard to calculate kidney function; however, recent research has proven that mGFR is not always superior to estimated GFR (eGFR). ${ }^{6}$ Estimated-Glomerular Filtration Rate (eGFR) is used by Kidney Disease: Improving Global Outcomes (KDIGO) to make new guidelines that can predict the prognosis of CKD.

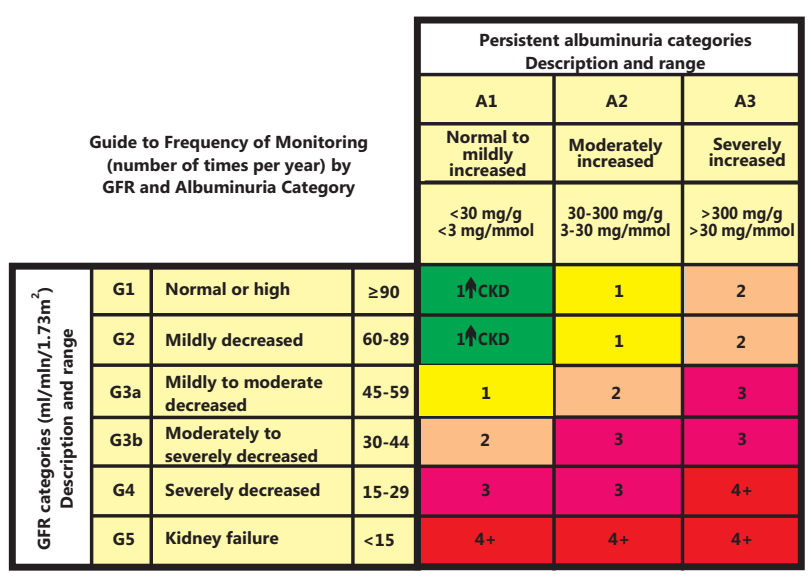

Figure 4. KDIGO guideline ${ }^{7}$

Green: low risk (absence of any other kidney marker); yellow: moderate risk; orange: high risk; red: very high risk

The eGFR in daily practice is measured indirectly (because the gold standard for measuring renal function is neither practical nor economical for daily use) using variables such as serum creatinine, age, gender, height, and weight for different formulas. ${ }^{6}$ One frequently used method is the calculation of creatinine clearance using the 24-hour urine, although this method is expensive, more complex, and improper collection of urine is frequently found.

Measurement of creatine clearance should be considered in circumstances when equation estimation based on serum creatinine is suspected to be inaccurate when highly accurate values are needed, and when exogenous markers to measure GFR is not available. This may occur in people who undergo evaluation for kidney donation or treatment with drugs with significant toxicity that are excreted by the kidneys (ex: high dose methotrexate), etc. Miscalculations often occur due to improper urine collection or over calculations of creatinine. ${ }^{6}$
This study showed that there were no significant differences from GFR using MDRD (sig 0.194) or CKD-Epi (sig 0.468). However, CG as the referral method of the American Diabetes Association showed a significant difference $(0.000) .^{8}$ This is because the CG formula has both glomerular and tubular creatinine clearance; therefore, sometimes there is an overestimation of value up to $40 \%$ in young individuals with no chronic kidney disease. This formula under-estimates the GFR value in elder individuals because age is included in the numerator of the CG formula (140 - age). Therefore, with the same weight and serum creatinine, elderly patients will have an unproportional GFR value., ${ }^{9,10}$

The CG formula estimates creatinine clearance but does not adjust the creatinine clearance for different body surface areas, whereas CKD-Epi and MDRD can calculate the kidney function despite the different sizes of the subjects. However, CG cannot be used for children; it underestimates creatinine clearance due to variation in age and weight. It is inaccurate when kidney function is unstable, kidney and liver failure, and is not standardized for body surface area. Cockroft-Gault is not accurate in a population that has factors that influence creatinine production, such as malnutrition, obesity, vegetarians, overly muscular people, elderly, amputated people, high consumption of meat, and creatinine-containing supplements, and fluctuated renal function. ${ }^{11}$

This research showed that MDRD results were not significantly different from CC, but the correlation was weaker than CKD-Epi, with a rho of 0.448 . Previous research suggested that MDRD is not accurate for populations without kidney disease, such as young patients with type 1 diabetes without microalbuminuria or patients being evaluated for kidney donation. ${ }^{12}$ Modification of diet in renal disease formula has not been validated for children (age < 18 years old), pregnant females, elderly patients ( $>85$ years old), or certain races, such as Hispanics. Modification of diet in renal disease limitations are also due to serum creatinine that has a relationship with nutritional status or therapy. ${ }^{6}$ The youngest and the eldest subject of this research respectively was 17 and 90 years old, this might influence the correlation of MDRD more than CG and CKD-Epi. ${ }^{6}$ Modification of diet in renal disease underestimates the GFR in Chinese people as much as $23 \%$ and overestimates GFR in the Japanese population up to $12 \%$. This discrepancy shows that MDRD needs to consider different races to detect and monitor kidney injury accurately, especially in Asia. ${ }^{13}$ 
This research showed that CKD-Epi was the right method for calculating eGFR, and this is suitable to the latest guideline by KDIGO CKD, which recommends CKD-Epi for estimating GFR unless another formula is proven to be better in a local population (Fig. 4). ${ }^{7}$ Jessani et al. who compared CKD-Epi and MDRD with inulin clearance as the gold standard, showed that CKD-Epi was significantly more accurate for the South Asian Population, even though both methods still overestimate GFR in the observed population. ${ }^{14} \mathrm{~A}$ study by Horio et al. also showed that CKD-Epi had a better performance than MDRD for eGFR in the South East Asian population. ${ }^{15}$

\section{CONCLUSION AND SUGGESTION}

There was a statistically significant correlation between the measurement of GFR using Creatinine Clearance with the 24-hour urine collection method, CG, MDRD, and CKD-Epi. This study showed that estimated GFR could be used as an alternative to GFR measurement with the 24-urine collection, which is harder, more expensive, and requires a longer time.

Further studies using an exogenous marker to determine the best formula to overview kidney damage were needed.

\section{REFERENCES}

1. Skorecki K, Greens J, Brenner B. Chronic renal failure. Harrisons principles of internal medicine. $16^{\text {th }}$ Ed., New York, Mc Graw Hill, 2005; 1653.

2. Crews DC, Bello AK, Saadi G. World kidney day editorial-burden, access, and disparities in kidney disease. J Bras Nefrol, 2019; 41(1): 1-9.

3. Jager KJ, Frase SDS. The ascending rank of chronic kidney disease in the global burden of disease study. Nephrol Dial Transplant, 2017; 32(suppl_2): ii121-ii128.

4. Gounden V, Bhatt $H$, Jialal I. Renal function tests. StatPearls Publishing. 2019. Available from: https:/www.ncbi.nlm,nih,giv/books/NBK507821 (accessed 20 September, 2020).

5. Florkowski CM, Janice Sc Chew-Harris. Methods of estimating GFR-different equations including
CKD-Epi. Clin Biochem Rev, 2011; 32(2): 75-9.

6. National Kidney Foundation. Frequently asked questions about GFR estimates. https://www.kidney. org/sites/default/files/12-10-4004 FAQ-ABE.pdf. 2017. (accessed 20 July, 2019)

7. Kidney Disease: Improving global outcomes (KDIGO) CKD work group. KDIGO 2012 clinical practice guideline for the evaluation and management of chronic kidney disease. Kidney Int Suppl, 2013; 3(1): 1-150.

8. American Diabetes Association. Standards of medical care for patients with diabetes mellitus. Diabetes Care, 2001; 24: S33-S43.

9. Ferreira JP, Girerd N, Pellicori P, Duarte K, Girerd S, et al. Renal function estimation and Cockcroft-Gault formulas for predicting cardiovascular mortality in population-based, cardiovascular risk, heart failure, and post-myocardial infarction cohorts: The Heart 'OMics' in AGEing (HOMAGE) and the high-risk myocardial infarction database initiatives. BMC Medicine, 2016; 14(1): 181.

10. Cartet-Farnier E, Goutelle-Audibert L, Maire P, De la Gastine B, Goutelle S. Implications of using the MDRD or CKD-Epi equation instead of the Cockcroft-Gault equation for estimating renal function and drug dosage adjustment in elderly patients. Fundam Clin Pharmacol, 2016; 31(1): 110-11.

11. Meredith C. Cockcroft Gault formula. Laboratory bulletin. Updates and information from Rex Pathology Laboratory, 2006; 121: 3-4.

12. Coresh J, Stevens LA. Kidney function estimating equations: Where do we stand?. Curr Opin Nephrol Hypertens, 2006; 15(3): 276-284.

13. Kearkiat Praditpornsilpa, Natavudh Townamchai, Tawatchai Chaiwatanarat, Khajohn Tiranathanagul, P Katawatin, et al. The need for robust validation for MDRD-based glomerular filtration rate estimation in various CKD populations. Nephrology Dialysis Transplantation, 2011; 26(9): 2780-2785.

14. Jessani $S$, Levey AS, Bux R, Inker LA, Islam $M$, et al. Estimation of GFR in South Asians: A Study from the general population in Pakistan. Am J Kidney Disease, 2014; 63(1):49-58.

15. Horio M, Imai E, Yasuda $Y$, Watanabe T, Matsuo S. Modification of the CKD epidemiology collaboration (CKD-Epi) equation for Japanese: Accuracy and use for population estimates. Am J Kidney Dis, 2010; 56: 32-38. 